\title{
Velocity and mass density of the ejecta produced from sinusoidal grooves in laser shock-loaded tin
}

\author{
G. PRUDHOMME, ${ }^{1}$ T. de RESSÉGUIER, ${ }^{2, \text { * }}$ C. ROLAND,,${ }^{1,2}$ \\ A. SOLLIER,${ }^{1,3}$ E. LESCOUTE,${ }^{1,3}$ D. LOISON, ${ }^{4}$ and E. BRAMBRINK ${ }^{5,6}$ \\ ${ }^{1}$ CEA, DAM, DIF, F-91297 Arpajon, FRANCE \\ ${ }^{2}$ Institut Pprime (UPR 3346), CNRS, ENSMA, Univ. Poitiers, F-86961 Futuroscope, FRANCE \\ ${ }^{3}$ Université Paris-Saclay, CEA, Laboratoire Matière en Conditions Extrêmes, F-91680 Bruyères-le-Châtel, FRANCE \\ ${ }^{4}$ Institut de Physique de Rennes, CNRS, Univ. Rennes 1, F-35042 Rennes, FRANCE \\ ${ }^{5}$ LULI, CNRS, École Polytechnique, CEA, Univ. Paris-Saclay, F-91128 Palaiseau, FRANCE \\ ${ }^{6}$ Sorbonne Universités, UPMC, Univ. Paris 06, CNRS, LULI, F-75252 Paris, FRANCE

\begin{abstract}
When a shock wave of several tens of GPa breaks out at a free surface, material is ejected ahead
The laser-based experiments presented in this paper focus on microjetting in shock-melted tin
\end{abstract} \\ of this surface. The amount and velocity of such ejecta depend on the breakout pressure, state of \\ the released material (solid, liquid, or mixed), whether the shockwave is supported or unsupported \\ and the initial geometrical perturbation (or roughness) of the free surface. If surface defects consist \\ of small grooves, pits or scratches, material ejection occurs in the form of jets breaking up into tiny \\ particles (so-called microjetting), with jet tip velocities up to several times higher than the free \\ surface velocity. \\ with periodic surface perturbations. Several complementary diagnostics are combined to measure the \\ velocity and mass of ejecta during the early stages of the jetting process. One relevant advancement \\ is the use of ps-laser X-ray radiography to probe the density of the ejecta in distinct jets a few tens \\ of $\mu \mathrm{m}$-wide. The effects of the depth and wavelength of the initial perturbation are investigated in \\ both linear and near-linear growth regimes. The results are compared with predictions derived from \\ the Richtmyer-Meshkov Instability (RMI) theory.
}

Published in Journal of Applied Physics, 128, 155903 (2020); https://doi.org/10.1063/5.0022940.

\section{INTRODUCTION}

The ejection of high velocity debris and shrapnel from materials and structures subjected to shock loading is a major safety issue for a variety of applications, including pyrotechnics [1, 2] or inertial confinement fusion in highpower laser facilities [3, 4. One of the processes governing such ejection is the interaction of a shock wave with a free surface presenting geometrical defects such as pits, scratches, grooves, or machining-inherited roughness. This process, usually referred to as "material ejection" or "microjetting" (because ejecta often consist of "jets" breaking up into micrometer-scale particles), has been the subject of extensive research work for about six decades [5], and it still motivates both theoretical and experimental investigations worldwide [6-14]. For a few years, we have explored how laser-driven shocks can provide some valuable, complementary insights into ejecta physics under pulsed pressure loads of high amplitude (some tens of GPa) and very short duration (a few ns). Our results include measurements of jet velocities using both optical shadowgraphy and Photonic Doppler Velocimetry (PDV) 15 17, post-recovery evidence of the jetting process combined with spall fracture [15, 17, 18, attempts to estimate ejecta size distributions using fast shadowgraphy [16, 19, 20, or fragment recovery [21, and ultra-fast laser-based X-ray radiography of the jets [17, 22, 23. The latter technique was used to evaluate mass ejection from single triangular grooves of controlled depths and angles [17. However, surface roughness resulting from machining processes is usually better represented by periodic perturbations. Besides, as will be recalled next, there is a wide agreement in the recent literature (e.g. Ref. 5 and references therein) that material ejection upon shock breakout can be considered as a limiting case of the Richtmyer-Meshkov Instability (RMI) theory [24, especially after partial or full melting of the shocked specimen. Still, isolated (non-periodic) grooves do not fully fit this picture because of inadequate boundary conditions at the groove edges [25. Furthermore, although Fourier analyses have been proposed to extend it to surface defects of various shapes [26, 27, this theory was initially developed for sinusoidal perturbations.

Here, we perform velocity measurements and laser-based X-ray radiography to investigate material ejection from laser shock-loaded tin samples, above melting conditions, with periodic grooves of approximately sinusoidal shape and controlled amplitudes and wavelengths in their free surface. The results are compared with data obtained from

* resseguier@ensma.fr 

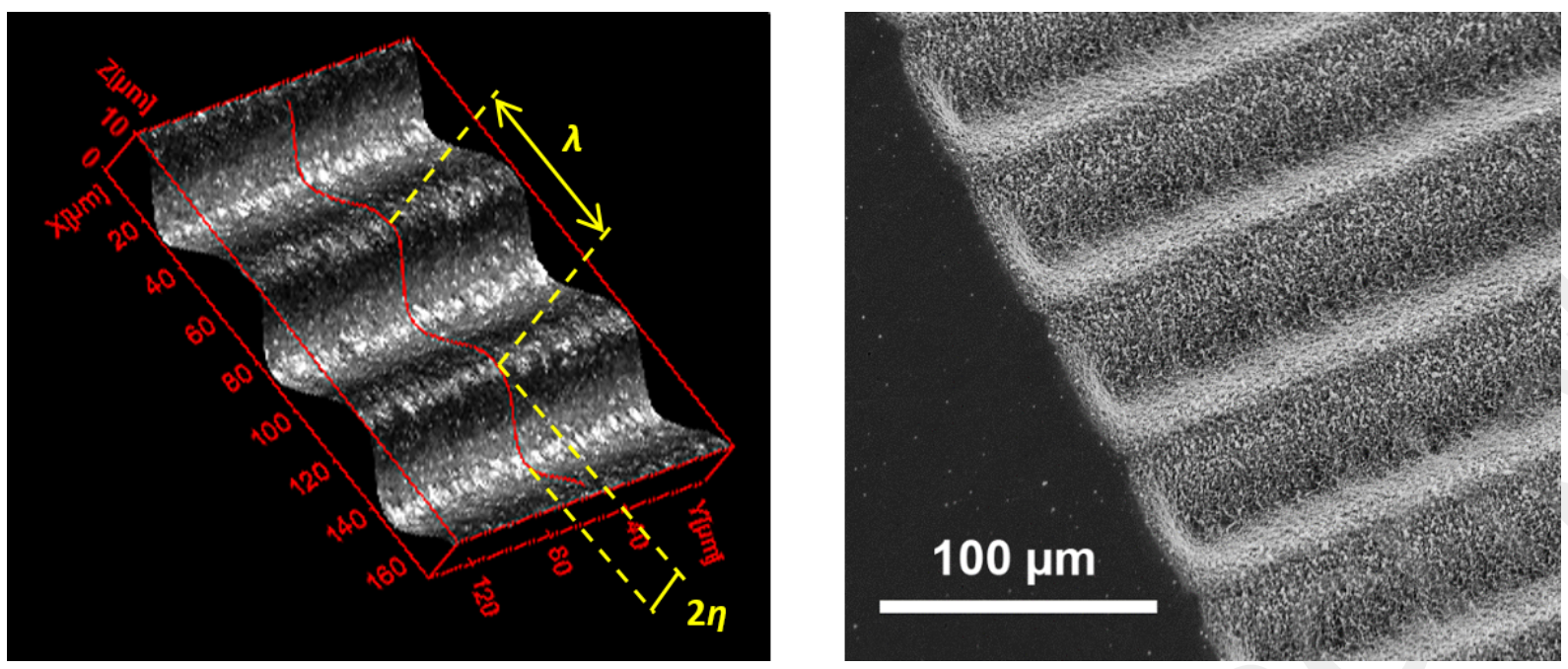

Figure 1. Typical pattern of periodic grooves engraved in the surface of the tin samples, reconstructed from optical micrographs (left) and observed in scanning electron microscopy (right). $2 \eta$ and $\lambda$ are the depth (peak-valley) and the wavelength of the grooves, respectively.

explosive-based experiments (involving much larger time scales), and they are used to test the predictive capabilities of RMI-based models in such specific loading conditions.

\section{EXPERIMENTAL SETUP}

The experiments were performed in the LULI large-scale laser facility (Laboratoire pour l'Utilisation des Lasers Intenses, École Polytechnique, France). Targets consisted of $230 \mu \mathrm{m}$-thick tin foils of $>99.7 \%$ purity, with grooved patterns of approximately sinusoidal shape engraved in their surface (Figure 1) using high frequency ablation by fs-laser pulses. Groove depth $2 \eta$ (peak-to-valley, see Figure 1) varied from about $7 \mu \mathrm{m}$ to $24 \mu \mathrm{m}$, and wavelength $\lambda$ was between $60 \mu \mathrm{m}$ and $180 \mu \mathrm{m}$. The corresponding $k \eta$ product, where $k=2 \pi / \lambda$, commonly used to study RMI-type ejection, ranges from about 0.4 to 1.2. Groove length was $1 \mathrm{~mm}$, and the width of the grooved pattern was as close to $1 \mathrm{~mm}$ as allowed by a whole groove number.

In the opposite surface (loaded side), a laser pulse of $1.06 \mu \mathrm{m}$ wavelength, $\sim 700 \mathrm{~J}$-energy, $\sim 5 \mathrm{~ns}$-duration was focused on a $1.5 \mathrm{~mm}$-diameter spot (Figure 2). The target was set in secondary vacuum to prevent laser breakdown in air. A thin absorbing layer is ablated into plasma, and its expansion towards the laser source drives by reaction a short compressive pulse in the solid sample. The compression front steepens into a shock while the unloading wave spreads, so that this pressure load becomes roughly triangular (so-called Taylor wave or unsupported shock) and peak pressure decays with propagation distance. When the shock wave breaks out at the opposite free surface, its interaction with the grooves produces the ejection of fast debris, in the form of jets ahead of the main, planar surface. Time-resolved measurements of both jets and planar surface velocities were performed using Photonic Doppler Velocimetry (PDV), while material ejection was observed in transverse optical shadowgraphy, using an ultra high-speed camera with a magnification of $1.93 \mathrm{\mu m} /$ pixel and exposure times of $5 \mathrm{~ns}$ to minimize motion blur [15-17. Finally, at a controlled delay time after the ns-drive, a 1.5 ps-duration laser probe of about $10 \mathrm{~J}$ was shot to the tip of a freestanding copper wire of $20 \mu \mathrm{m}$-diameter set about $1 \mathrm{~cm}$ below the grooved pattern. Subsequent emission of a ps-duration X-ray pulse, mainly K-shell radiation (at $8 \mathrm{keV}$ for copper), allows ultra-short in-situ radiography of the ejecta [17, 22, 23] along the third (vertical) direction, normal to the shadowgraphy axis (Figure 2), using a distant image plate shielded with a $15 \mu \mathrm{m}$-thick aluminum foil. The spatial resolution was evaluated at about $20 \mu \mathrm{m}$ from a static radiograph of a metal grid set at the sample position [22]. On-shot calibration of the X-ray signal was obtained from tin step wedges of controlled thicknesses inserted in front of the image plate, providing a direct correlation between the photo-stimulated luminescence recorded on the image plate and areal mass density. More details on the geometry as well as an example of X-ray spectrum (monitored at each shot inside the vacuum chamber) can be found in Ref. 17. Lasers and diagnostics are synchronized using optical marks, where the time resolution is limited by the bandwidth of the slowest photodiode $(\approx 500 \mathrm{MHz})$. 

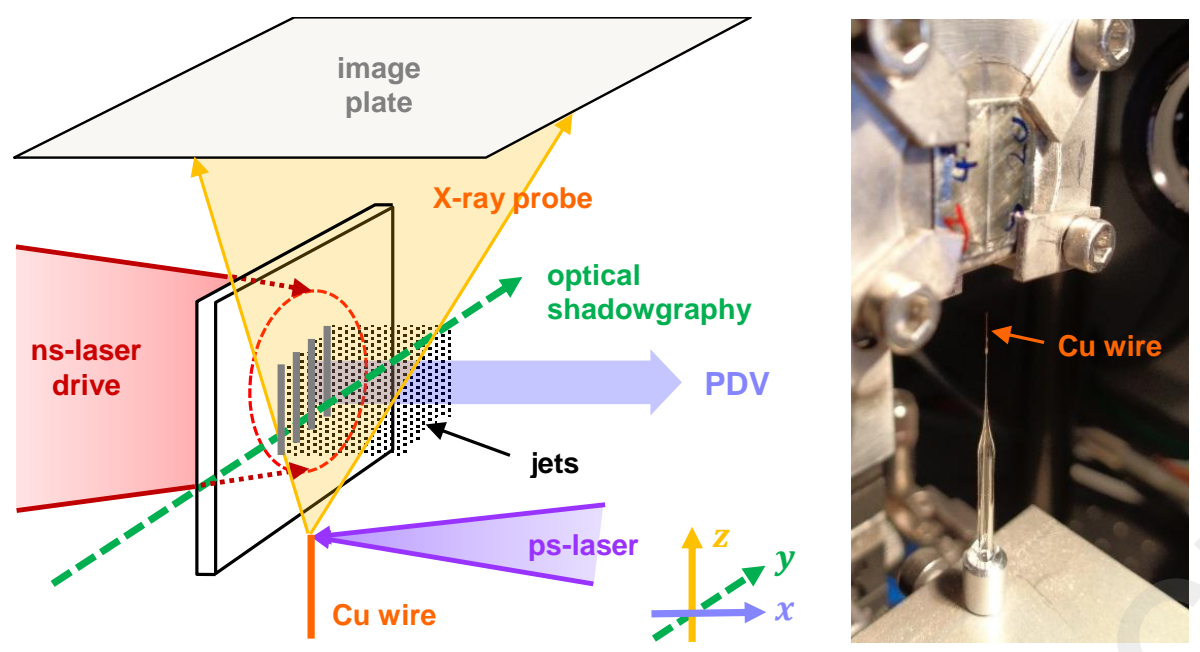

Figure 2. Schematic of the setup (left). Irradiation by a ns-laser pulse drives a shock in a tin sample. Shock breakout at the opposite free surface produces micro-jetting from a grooved pattern. Free surface velocity is measured using Photonic Doppler Velocimetry (PDV), while optical shadowgraphy allows transverse observation of jet expansion. At a controlled delay time after shock loading, a ps-laser pulse is focused to the tip of a freestanding copper wire of 20 um-diameter (right picture, barely visible) set about $1 \mathrm{~cm}$ below the grooved pattern (actually a single vertical groove in this picture). Subsequent emission of a ps-duration X-ray probe allows ultra-short radiography of the ejecta along the third (vertical) direction.

\section{EXPERIMENTAL RESULTS}

The main experimental parameters and results are listed in Table I.

Table I. Summary of the main experimental parameters and results. $v_{\mathrm{fs}}$ is the free surface velocity outside the grooved pattern, $v_{\mathrm{jt}}$ is the jet tip velocity, both inferred from shadowgraphy. $P_{\mathrm{SB}}$ is shock breakout pressure, calculated from $v_{\mathrm{fs}}$. $t_{\mathrm{rad}}$ is the time delay between shock breakout and X-ray radiography. $M_{\mathrm{ej}}$ is the total areal mass ejected from the grooves. $v_{99 \%}$ is the velocity of a front containing $99 \%$ of this ejected mass.

\begin{tabular}{|c|c|c|c|c|c|c|c|c|}
\hline Id & $v_{\mathrm{fs}}[\mathrm{km} / \mathrm{s}]$ & $P_{\mathrm{SB}}[\mathrm{GPa}]$ & $k \eta$ & $\lambda[\mu \mathrm{m}]$ & $t_{\mathrm{rad}}[\mathrm{ns}]$ & $v_{\mathrm{jt}}[\mathrm{km} / \mathrm{s}]$ & $v_{99 \%}[\mathrm{~km} / \mathrm{s}]$ & $M_{\mathrm{ej}}\left[\mathrm{mg} / \mathrm{cm}^{2}\right]$ \\
\hline Sn06 & 2.50 & 41.7 & 0.38 & 60 & 360 & 4.85 & 4.36 & 6 \\
\hline $\mathrm{Sn} 08$ & 2.76 & 47.9 & 1.23 & 60 & 360 & 6.61 & 5.60 & 12 \\
\hline Sn10 & 2.59 & 43.6 & 0.42 & 120 & 359 & 6.21 & 4.92 & 15 \\
\hline Sn14 & 2.74 & 47.2 & 0.40 & 120 & 560 & 6.26 & 4.39 & 17 \\
\hline Sn16 & 2.79 & 48.5 & 0.55 & 60 & 360 & 5.13 & 4.48 & 7 \\
\hline Sn17 & 2.61 & 44.3 & 0.56 & 60 & 160 & 4.88 & 4.76 & 10 \\
\hline Sn21 & 2.77 & 48.0 & 0.50 & 96 & 360 & 5.75 & 5.07 & 10 \\
\hline Sn25 & 2.60 & 44.0 & 0.38 & 60 & 160 & 5.12 & 4.34 & 6 \\
\hline $\operatorname{Sn} 27$ & 2.82 & 49.2 & 0.41 & 180 & 359 & 7.78 & 5.73 & 16 \\
\hline Sn32 & 3.04 & 54.7 & 0.41 & 180 & 160 & 6.47 & - & - \\
\hline Sn12 & 2.62 & 44.4 & 0.40 & 60 & 360 & 4.10 & 4.12 & 16 \\
\hline Sn30 & 2.64 & 44.8 & 0.41 & 60 & 160 & 4.49 & - & - \\
\hline
\end{tabular}

\section{A. Free surface velocity and shock breakout pressure}

As reported in previous experiments [15, 17] and recalled above, the velocity of the planar free surface outside the grooved patterns can be determined using transverse optical shadowgraphy and/or PDV. Figure 3 shows a PDV spectrogram recorded behind a laser shock-loaded tin sample with a single, laser-engraved groove in its free surface (experiment Sn12). It is very similar to those reported in Ref. 17 for single triangular grooves. From the raw PDV signal, where time origin is the ns-laser shot, shock breakout at the free surface is found to occur 41 ns after this shot. In the spectrogram, the main line at $2.83 \mathrm{~km} / \mathrm{s} \pm 60 \mathrm{~m} / \mathrm{s}$ is the planar free surface velocity outside the groove, while faint traces above this line probably come from particles in the micro-jet expanding from the groove. However, in all 

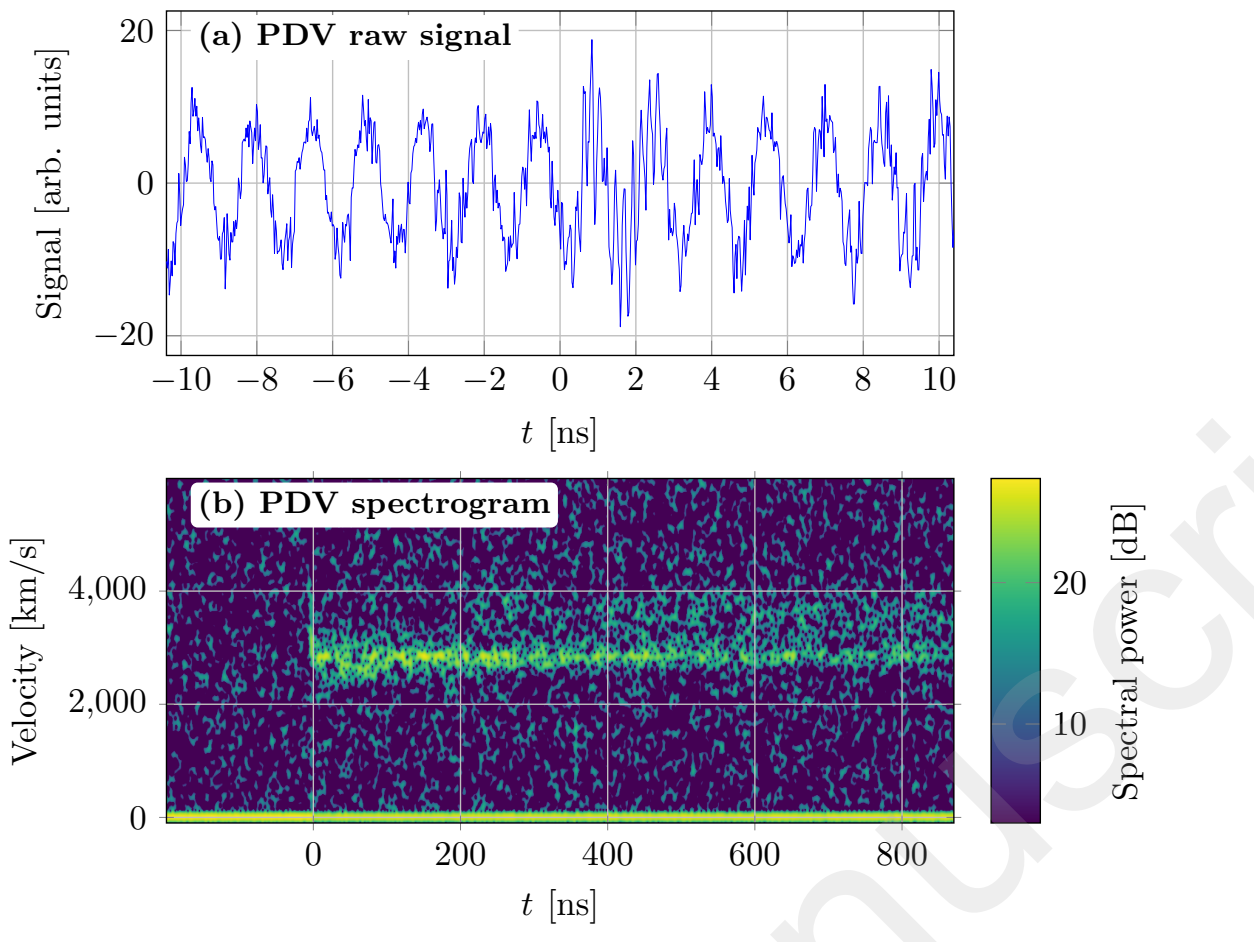

Figure 3. PDV record behind a laser shock-loaded tin sample with a single groove in its free surface (Sn12). Data are processed using a 17 ns (zero to zero) Hann sliding window. Time origin coincides with the shock breakout. (a) PDV raw signal, (b) PDV spectrogram.

the shots involving periodic grooves, where the size of the grooved pattern, about $1 \mathrm{~mm}^{2}$, was close to the diameter of the collimated PDV probe, only very weak return signals could be detected, too weak to extract a surface velocity from the background noise. We attribute this weakness to the loss of overall reflectivity upon shock breakout, due to extensive micro-jetting from the grooves, while pre-shot adjustment of the recording parameters was based on the intact, highly reflective sample surface.

In the shadowgraphs recorded in the same shot (similar to Figure 4 further), the position of the planar free surface outside the groove was observed to increase linearly with time, within a $0.9996 \mathrm{R}$-square fit, between $75 \mathrm{~ns}$ and $650 \mathrm{~ns}$ after shock loading, indicating a constant velocity of $2.71 \mathrm{~km} / \mathrm{s}$, in very good agreement with the PDV measurement. Thereafter, this shadowgraphy-based method was used to evaluate the free surface velocity in all shots. The results were found to lie within $\pm 140 \mathrm{~m} / \mathrm{s}$ around a mean value of about $2.7 \mathrm{~km} / \mathrm{s}$ (Table I). Scatter is likely due to shot-to-shot variations of the drive intensity, mainly caused by laser energy fluctuations.

According to a common approximation, the particle velocity behind the shock just before it reaches the free surface is $u_{\mathrm{SB}} \approx \frac{1}{2} v_{\mathrm{fs}}$. Hugoniot conservation equations then provide shock breakout pressure $P_{\mathrm{SB}}=\rho_{0} D_{\mathrm{SB}} u_{\mathrm{SB}}$, where $\rho_{0}=7.29 \mathrm{~g} / \mathrm{cm}^{3}$ is tin density and $D_{\mathrm{SB}}=c_{0}+s \times u_{\mathrm{SB}}$ is the shock velocity, with $c_{0}=2.61 \mathrm{~km} / \mathrm{s}$ and $S=1.49$ [28]. The mean value of $46 \mathrm{GPa}$ is much higher than the 20-32 GPa range where tin is known to melt upon release [eg., 24, 29, 30, so that the ejection process in these experiments is expected to occur in a fully liquid state.

\section{B. Ejection Velocity}

Like that of the planar free surface, the position of the front of the debris cloud expanding in vacuum from the grooved pattern is found to increase linearly with time in the shadowgraphs 25 . This provides the jet tip velocity $v_{\mathrm{jt}}$ (also known as spike velocity) for each shot, within an experimental uncertainty estimated to $\pm 5 \%$, mainly due to the imperfect planarity of the front and its increasing fuzziness at late times (Figure 4).

The results are plotted in Figure 5. As mentioned earlier in Subsection III A the velocity of the planar free surface is about the same for all shots. Jet tip velocity is found to increase with the $k \eta$ product characterizing the geometrical perturbation, which is consistent with both previous experiments and theoretical predictions. However, it also seems to strongly increase with the perturbation wavelength $\lambda$, as clearly illustrated in Section 4 showing two sequences recorded for similar $k \eta$ products but different wavelengths. Finally, the ratio of jet tip velocity to free surface velocity 

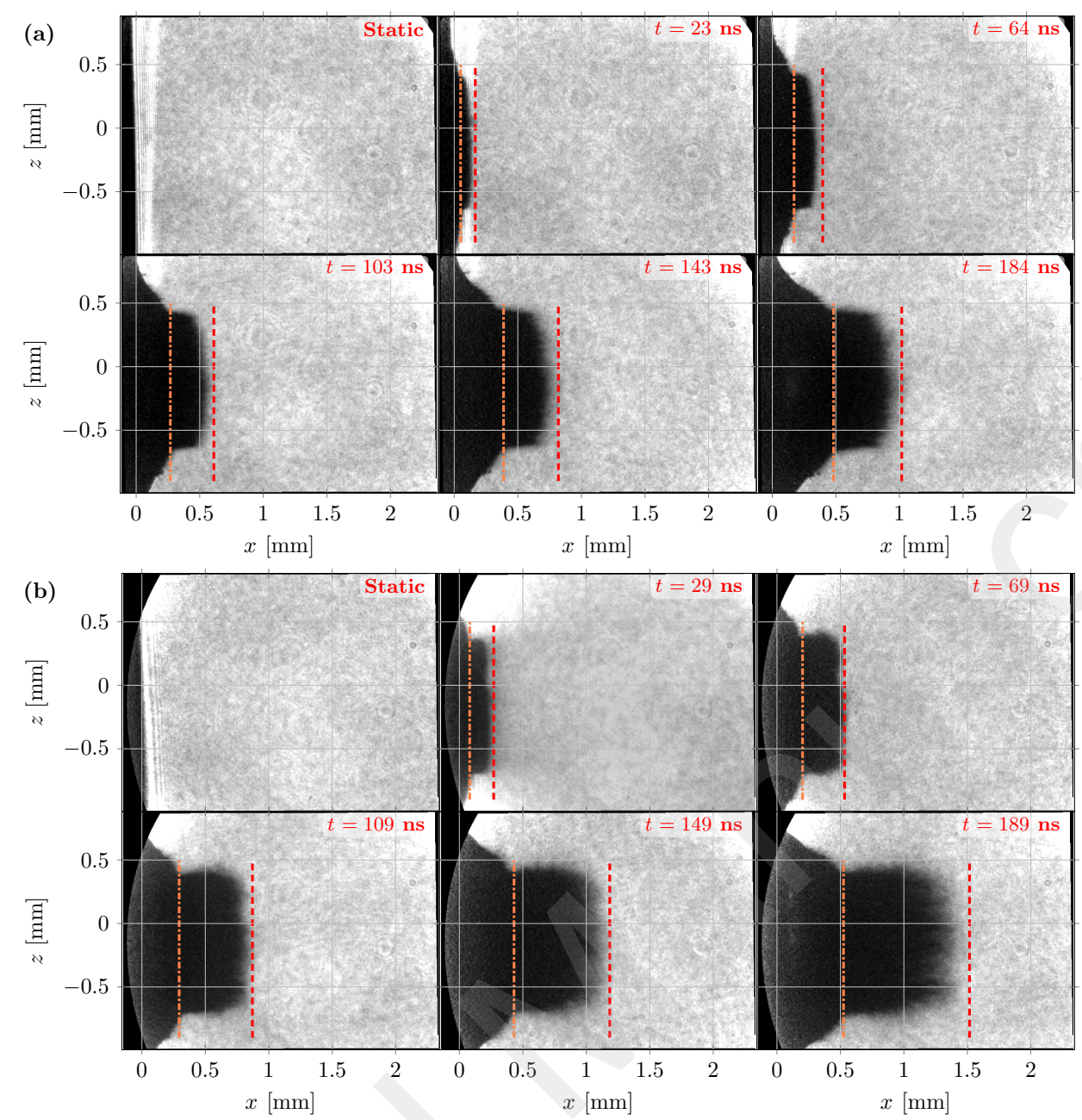

Figure 4. Optical shadowgraphs recorded behind laser shock-loaded tin samples in shots Sn25 (a) and Sn27 (b), showing the expansion (to the right) of ejecta clouds from grooved patterns of similar $k \eta \approx 0.4$ and wavelength $\lambda=60 \mu \mathrm{m}$ (a) or $180 \mu \mathrm{m}$ (b), as well as the motion of the smooth planar surface outside these patterns (peripheral zone). Time since shock breakout is inserted in each frame.

is found to reach surprisingly high values up to about 3. All this will be discussed in detail in Section IV

\section{Ejected mass}

A single X-ray image is taken for each shot. Nevertheless, the accurate control of the delay time between the ns-laser drive and the ps-laser probe allows imaging different stages of the ejection process, actually early stages in the present work, i.e. within the first $\sim 600 \mathrm{~ns}$ after shock breakout (see Table I).

As stated earlier in Section III a set of tin stepwedges is placed in the X-ray field of view in order to estimate the relationship between the equivalent tin thickness and the attenuation. This thickness is quantified as a transversal surface mass (which is not the areal mass of ejecta) using tin density. As illustrated in Ref. 17 the spectrum associated with our laser-generated X-ray probe shows an intense peak at $8 \mathrm{keV}$ matching the K-shell emission from copper, but it also reveals the significant contribution of continuous radiation, so that the source cannot be considered as fully monochromatic. Therefore, the attenuation was approximated with a double exponential fit:

$$
I\left(\rho_{0} \ell\right)=\frac{I_{0}}{2}\left(e^{-\mu_{1} \cdot \rho_{0} \ell}+e^{-\mu_{2} \cdot \rho_{0} \ell}\right)+I_{\mathrm{dark}}
$$

Where $I_{\text {dark }}$ is the dark-field level without direct X-ray exposures. Figure 6 illustrates such fit of experiment Sn06. The main uncertainty is related to the standard deviation in foil thickness, provided by the supplier, leading to the horizontal error bars. 


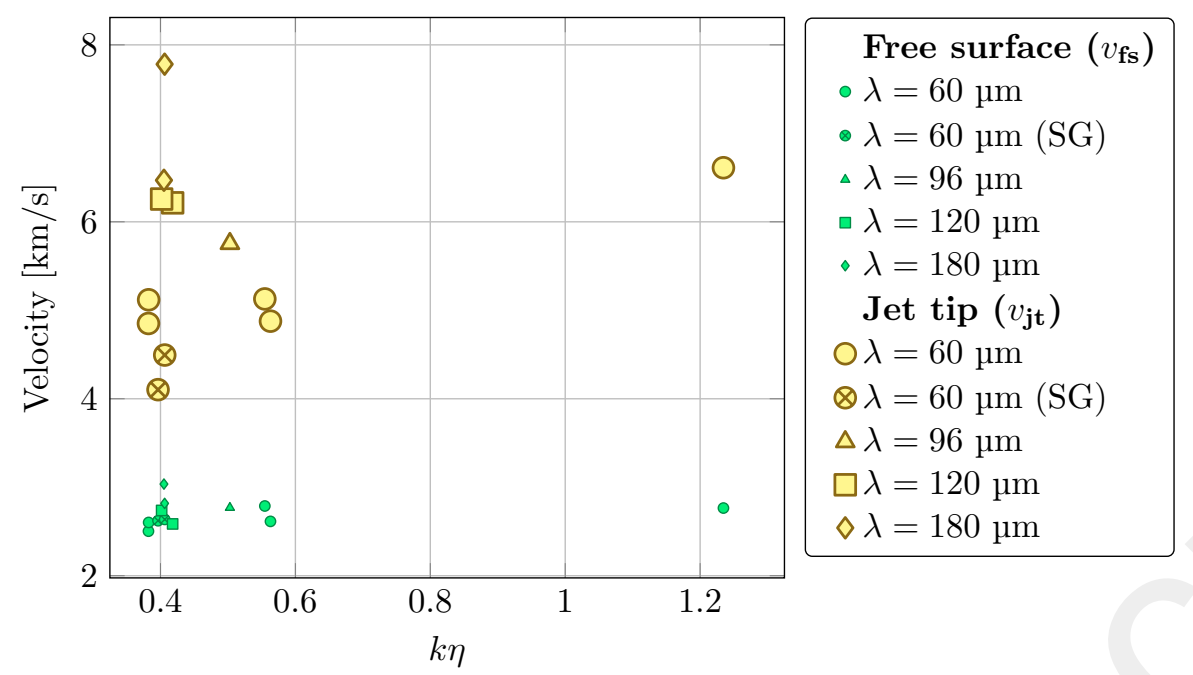

Figure 5. Free surface velocities and jet tip velocities inferred from the optical shadowgraphs for different surface perturbations (see text for details). SG stands for single groove.

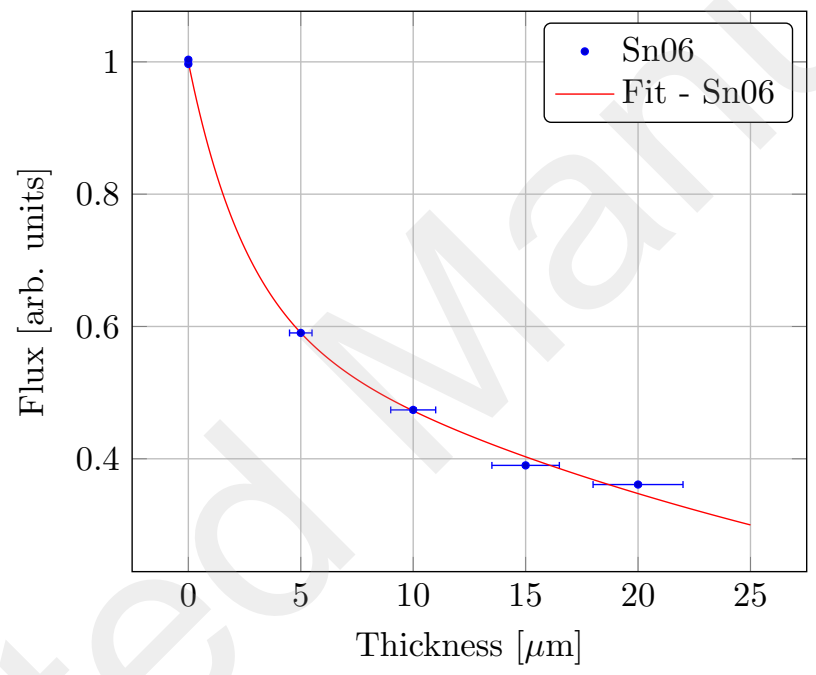

Figure 6. Typical fit of the relationship between the equivalent tin thickness and the X-ray attenuation (experiment Sn06).

Radiographs are analyzed through successive steps detailed below and illustrated in Figure 7.

- Raw image is corrected from irradiance inhomogeneity (see Appendix A, and Figure 7, left).

- The region-of-interest (ROI) around the ejecta is cropped and converted to an equivalent tin thickness $\ell$, using the correlation based on the stepwedges as explained above (Figure 7, top right).

- Next, a double integration along transverse $(y)$ and ejection $(x)$ directions gives the total cumulated mass. Finally, the areal mass $M s$ is obtained by dividing the total mass by the ejecting surface, i.e. the product of the vertical size $(\Delta y)$ of the ROI and the length of the grooved pattern in the groove direction $(\Delta z, 1 \mathrm{~mm}$ in our experiments):

$$
M_{\mathrm{S}}(x)=\frac{1}{\Delta z} \int_{x}^{x_{\mathrm{jt}}}\left(\frac{1}{\Delta y} \int \rho_{0} \ell(x, y) \mathrm{d} y\right) \mathrm{d} x
$$

Using $\Delta z=1 \mathrm{~mm}$, we assume that the jets are uniform in density side-to-side at the time of radiograph. Although the contours of the jets, which can be observed in the shadowgraphs in the $x$ - $z$ plane, seem to match this $1 \mathrm{~mm}$-length (Figure 4 for instance), possible variations of density along the $z$ direction have not been checked in our experiments. 

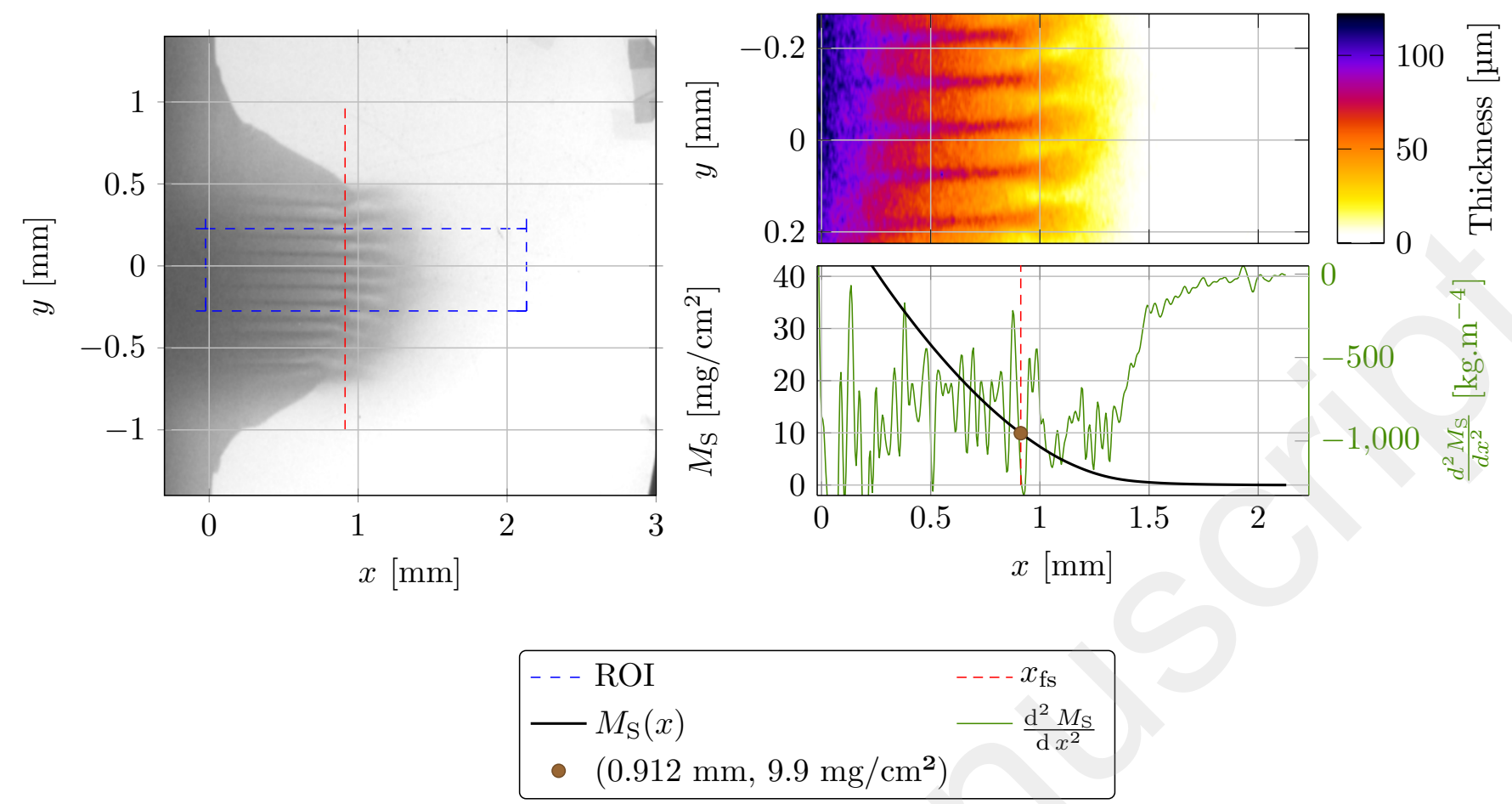

Figure 7. Analysis of a typical radiograph (experiment $\operatorname{Sn} 21, \lambda=96 \mu \mathrm{m}, t_{\mathrm{rad}}=360 \mathrm{~ns}$ ). Left: radiograph, with the region-ofinterest (blue frame) and the position of the free surface $x_{\mathrm{fs}}$ (red line); top right: conversion to the equivalent tin thickness $\ell$; bottom right: areal mass $M_{\mathrm{S}}$ along the ejection direction $x$ (black line), with its second derivative (green line).

The total areal mass in the jet $M_{\mathrm{ej}}$ at the probed instant is given by the value of $M_{\mathrm{S}}$ at the position $x_{\mathrm{fs}}$ of the free surface (red dotted line) outside the grooved pattern (Figure 7, bottom right).

$M_{\mathrm{ej}}$ is very dependent on the value of $x_{\mathrm{fs}}$, due to the important density increase near the free surface location; the estimation given by shadowgraphy $\left(\hat{x}_{\mathrm{fs}}=v_{\mathrm{fs}} \cdot t_{\mathrm{rad}}\right)$ is not precise enough to get reliable results. An analysis detailed in Appendix $\mathrm{B}$ based on the second derivative $\frac{\mathrm{d}^{2} M_{\mathrm{S}}}{\mathrm{d} x^{2}}$ improves the determination of $x_{\mathrm{fs}}$. This criterion is more objective than a visual estimation of the curve inflection.

Our experiments give access to parametric study of the microjetting phenomenon. All radiographs show similar features:

1. A fuzzy front at the leading edge of the ejecta cloud, with density fluctuations along the transverse direction (y) visible for the larger wavelengths. The jet tip position inferred from the optical shadowgraphs is inserted in most pictures as an orange mixed line. It is found to be much further than the cloud front in the radiograph. This is due to a major difference in sensitivity between both techniques, as will be discussed further.

2. Ahead of the free surface, distinct jets expanding from each groove. Ejecta density increases from the jet tip to the free surface, represented as a red dotted line inserted in the radiographs like in Figure 7

3. Parallel lines of low density growing from the grooves into the bulk (to the left), deep below the free surface. Such low-density regions are too deep to be associated with the "bubble growth" involved in the RMI theory. Instead, they are attributed to "cavitation waves", similar to those which were observed at the edges of grooved patterns under explosive loading 24, 31, 32, due to the interaction of the rarefaction waves reflected from the outer groove edge and from the planar surface outside the grooved area. In our case, the rarefaction waves coming from the grooves all interact with the incident unloading wave which immediately follows the shock front due to the very short duration of the pressure load, typically shorter by two orders of magnitude than in explosive-based experiments [17, 25]. Thus, such extensive interaction of release waves within the liquid metal produces cavitation not only at the outer edge of the grooved pattern but below each individual groove, as also observed in 3D simulations [1].

4. A lateral, outward deviation of the jets over a short distance near the position of the free surface, more pronounced at the periphery of the loaded region and at late times. We attribute such apparent deviation to 


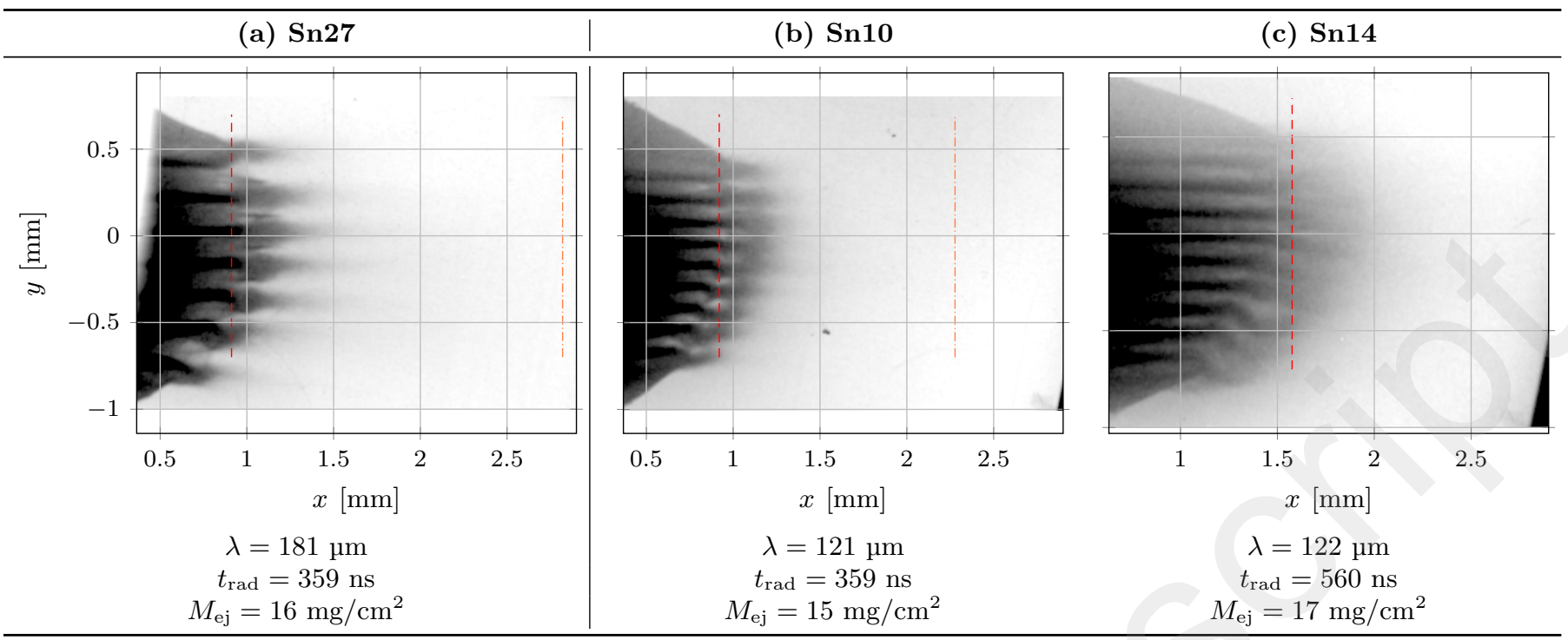

Figure 8. Effects of time and wavelength in three experiments, Sn27 (a), Sn10 (b) and Sn14 (c) sharing the same shape factor $k \eta \approx 0.41$. Red dashed and orange dot-dashed are respectively free surface $\left(x_{\mathrm{fs}}\right)$ and jet position $\left(x_{\mathrm{jt}}\right)$ inferred from the corresponding shadowgraphs.

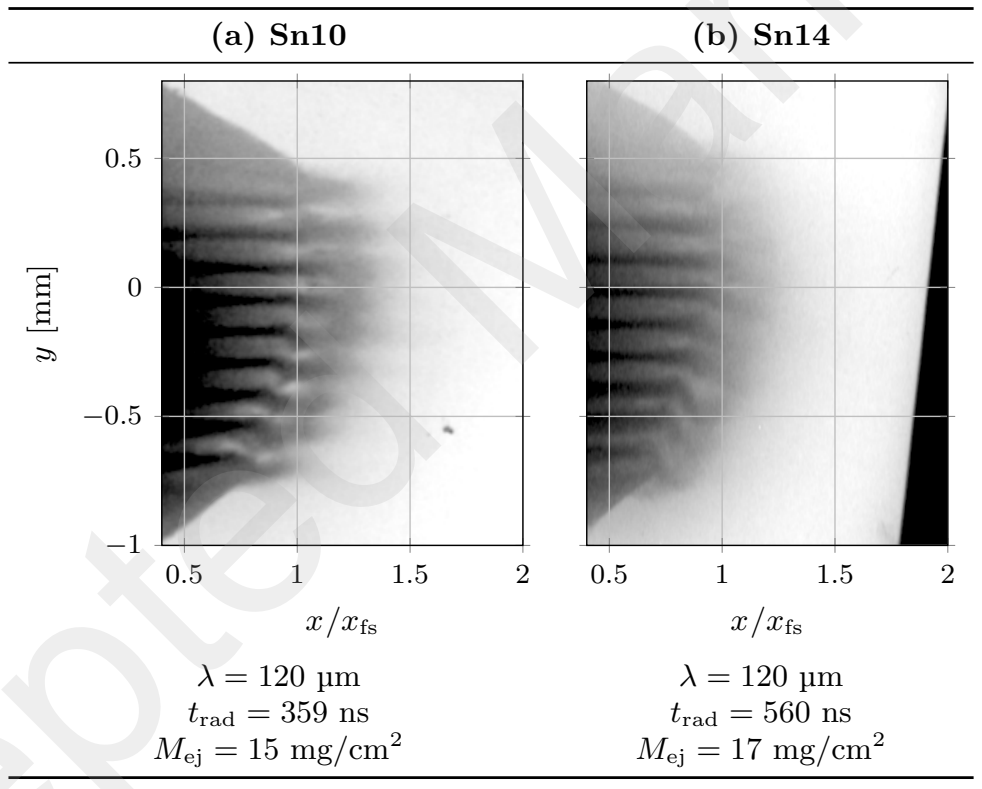

Figure 9. Stationarity of features for two different radiographs at time $t_{\mathrm{rad}}$. Distance along the $x$-axis is divided by $x_{\mathrm{fs}}$. Both experiments share the same shape factor $k \eta \approx 0.41$ at time $t_{\mathrm{rad}}=359 \mathrm{~ns}(\mathrm{a})$ or $t_{\mathrm{rad}}=560 \mathrm{~ns}(\mathrm{~b})$.

the overall curvature of the sample at the time of the radiograph. While both jets and cavitation waves have expanded from the initial grooves, leaving straight parallel lines on both sides of the free surface, radiography through the curved shell that the target has become shows local changes in directions resulting from late rotation (see e.g. Figure 8b or Figure 10a).

Figures 7 and 8 illustrate the effects of both perturbation wavelength and radiograph time. Thus, between Figures 7. 8b and 8 a , the ejected mass is found to increase $\left(10,15\right.$ and $\left.16 \mathrm{mg} / \mathrm{cm}^{2}\right)$ with the wavelength $(96,120$ and $180 \mu \mathrm{m})$. Besides, for identical grooves, the ejected mass increases from 15 to $17 \mathrm{mg} / \mathrm{cm}^{2}$ between $359 \mathrm{~ns}$ and $560 \mathrm{~ns}$ from shock breakout (Figure 8b and Figure 8k). In Figure 9, these two radiographs are compared along scaled, adimensional distance, i.e. $x$ was divided by $x_{\mathrm{fs}}$. Similar sizes and locations of all features in both images assess the stationarity of the micro-jetting process over this time range. The latest image appears to be more blurred, due to the increase of 
parallax and jet stretching.

According to shadowgraph Sn14 (Figure 8k), some of the particle cloud goes beyond the radiograph field of view due to the late $t_{\mathrm{rad}}\left(x_{\mathrm{jt}}=3.60 \mathrm{~mm}\right.$ whereas the maximum $x$ is $\left.2.8 \mathrm{~mm}\right)$. However, the $\mathrm{X}$-ray attenuation is very weak in this area $(12 \%)$, so the missing mass outside the field is estimated to be less than $6 \%$ of the total ejected mass. No other experiment misses particles outside X-ray field of view.

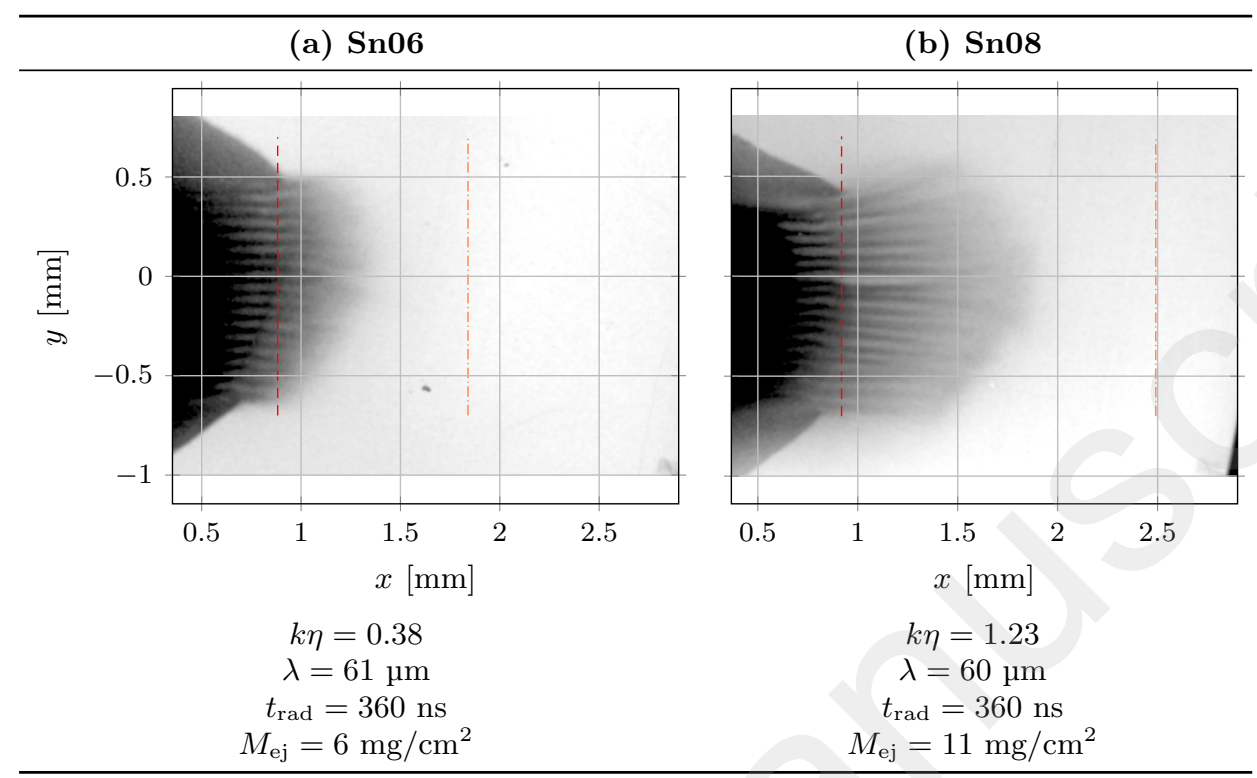

Figure 10. Effect of $k \eta$ on material ejection for experiments Sn06 (a) and Sn08 (b).

The $k \eta$ product characterizing the geometric perturbation is known to have a key influence on material ejection. This effect is clearly illustrated in Figure 10, showing the major increase of ejected mass $\left(\right.$ from 6 to $\left.11 \mathrm{mg} / \mathrm{cm}^{2}\right)$ with $k \eta(0.38$ to 1.23$)$.
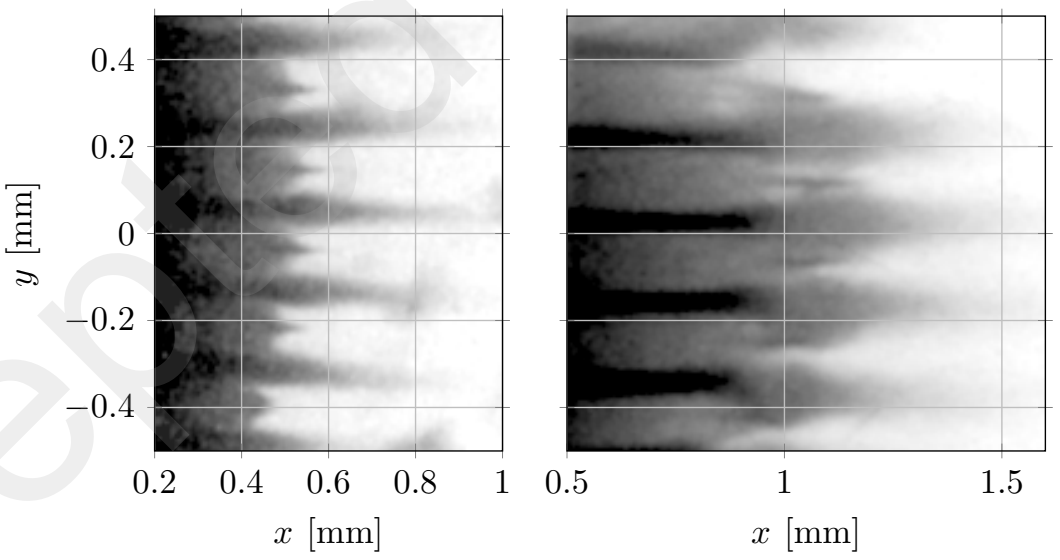

Figure 11. Evidence of second order harmonics between main jets: experiments Sn32 (left, $\lambda=180 \mu \mathrm{m}, k \eta=0.41, t_{\mathrm{rad}}=160 \mathrm{~ns}$ ) and $\operatorname{Sn} 27$ (right, $\lambda=180 \mu \mathrm{m}, k \eta=0.41, t_{\mathrm{rad}}=360 \mathrm{~ns}$ ).

Finally, Figure 11 shows two radiographs recorded at the largest wavelength of 180 um. Unfortunately, they could not be precisely converted to mass density, due to an attempt to insert a new X-ray filter in the setup. So, no ejecta mass are provided for these two experiments in Table I. However, they reveal secondary jets between main jets. They might be attributed to the non-linear growth of instability as described in Ref 33 . On the other hand, the relatively low value $k \eta=0.41$ would rather suggest linear growth. At a later time (Sn27), similar secondary jets are still visible at $y=0.1 \mathrm{~mm}$ and $y=0.3 \mathrm{~mm}$. In other inter-jets spaces, it is possible that secondary jets collide with primary ones, as strongly suggested by this Sn27 radiograph. Some molecular-dynamics or hydrodynamics simulations 27 revealed secondary jets for sinusoidal and chevron initial perturbations, but their relative amplitudes were not as large 

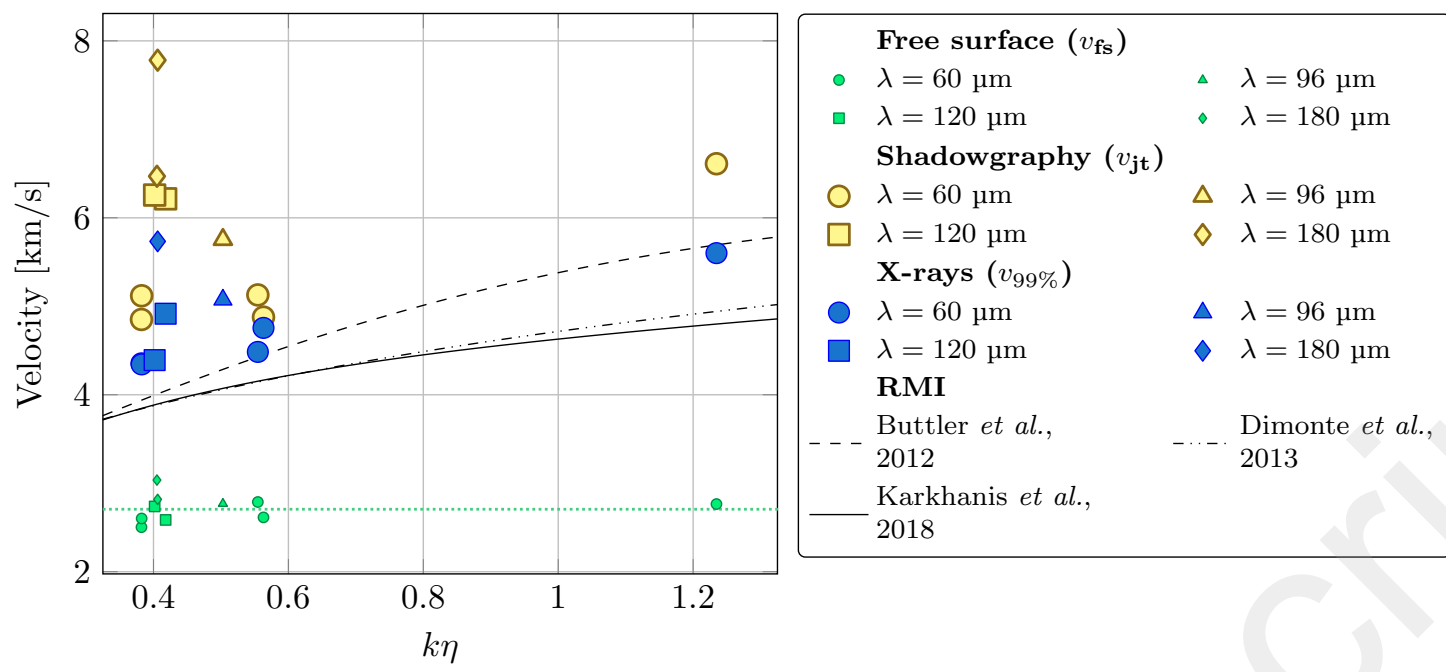

Figure 12. Comparison of the jet tip velocities inferred from the shadowgraph $\left(v_{\mathrm{jt}}\right.$, same as Figure 5), those resulting from the $99 \%$ mass criterion $\left(v_{99 \%}\right.$, see text), and those predicted by the Richtmyer-Meshkov Instability theory and models. A shock breakout pressure of $46 \mathrm{GPa}$ inferred from the average free surface velocity of $2.7 \mathrm{~km} / \mathrm{s}$ (green dotted line) was used in the RMI equations.

as observed in Figure 11. Alternatively, the initial surface perturbation for such deep grooves was observed to deviate locally from an ideal sinusoidal shape, as will be discussed in the next Section, which might create harmonics with smaller wavelengths. A third possible explanation could be secondary jetting after inversion of the initial perturbation, upon arrival of a reloading wave following a full transit of the reflected pulse back to the irradiated surface and forth to the free surface again. However, extensive cavitation is expected to occur during unloading in the liquid state (socalled micro-spall [30]), so that further compression by such reloading wave and subsequent jetting from the broken up surface would probably take place in very different conditions.

\section{DISCUSSION}

According to the RMI prediction, jet tip velocity is expected to increase with the $k \eta$ product characterizing the geometrical perturbation in the initial free surface, for a given value of shock breakout pressure [24, 34, 35]:

$$
v_{\text {spike }}=v_{\mathrm{fs}}+F_{\infty}^{s} F_{n l}^{s} F_{l} k \eta v_{\mathrm{fs}}
$$

where $F_{l}$ and $F_{n l}^{s}$ are linear and non-linear correction factors for spikes depending on $k \eta$ and $v_{\mathrm{fs}}$ as summarized in Ref. 24, and where $F_{\infty}^{s}$ was introduced to account for compressibility and asymptotic non-linear effects (while $F_{\infty}^{s}=\sqrt{3}$ in Ref. 24, considering a linear regime, i.e. $\left.k \eta \ll 1\right)$. Next, we retain two refined expressions of $F_{\infty}^{s} F_{n l}^{s}$, proposed by Dimonte et al. [34] and Karkhanis et al. [36]. Both were tested by their authors using molecular-dynamics or hydrodynamic simulations. In particular, it was suggested from these fits that a robust criterion to track the spike tip would be to consider a mass fraction threshold value of $1 \%$, i.e. the jet tip should be assimilated to the front of a cloud containing $99 \%$ of the ejected mass. Therefore, we have determined the position of this front in our radiographs (see Subsection III C), and derived its mean velocity $v_{99}$ between shock breakout and the radiograph instant.

A comparison between RMI spike velocity (Equation 3) with three different expressions of $F_{n l}^{s}$, by Buttler et al. 24, Dimonte et al. 34 and Karkhanis et al. [36, the jet tip velocity inferred from the shadowgraphs $\left(v_{\mathrm{jt}}\right.$, same as Figure 5), and that resulting from the $99 \%$ mass criterion $\left(v_{99 \%}\right)$ is plotted in Figure 12. Expectedly, the experimental values $v_{99 \%}$ compare much better with the RMI models than $v_{\mathrm{jt}}$. It should be pointed out that the large differences between $v_{\mathrm{jt}}$ and $v_{99 \%}$ are due not only to the removal of the fastest $1 \%$ mass at the jet tip, as stated above, but also, and mainly, to the higher sensitivity of optical shadowgraphy than X-ray radiography to detect small numbers of tiny particles at the very tip of the jet, not dense enough to significantly absorb the X-ray probe. This leads to a large shift between the leading edge of the clouds in the radiographs and the jet tip position inferred from the shadowgraphs (orange lines in Figures 8a, 8b, 10a). Ejection velocity seems to increase with the wavelength $\lambda$, which does not fit the RMIbased prediction. The overall match seems better with Buttler et al. 24, which would suggest that accounting for compressibility does not improve the description in our experimental conditions. The $v_{99 \%} / v_{\mathrm{fs}}$ ratio ranges between about 1.2 and 2 for all wavelengths, which is consistent with values reported in the literature. 


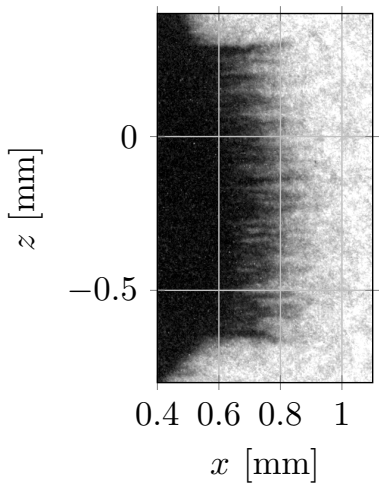

Figure 13. Shadowgraph recorded $163 \mathrm{~ns}$ after shock breakout behind a free surface containing one single groove (experiment Sn12). The non-uniformity of ejection along the groove direction (vertical in the picture) is clearly evidenced.

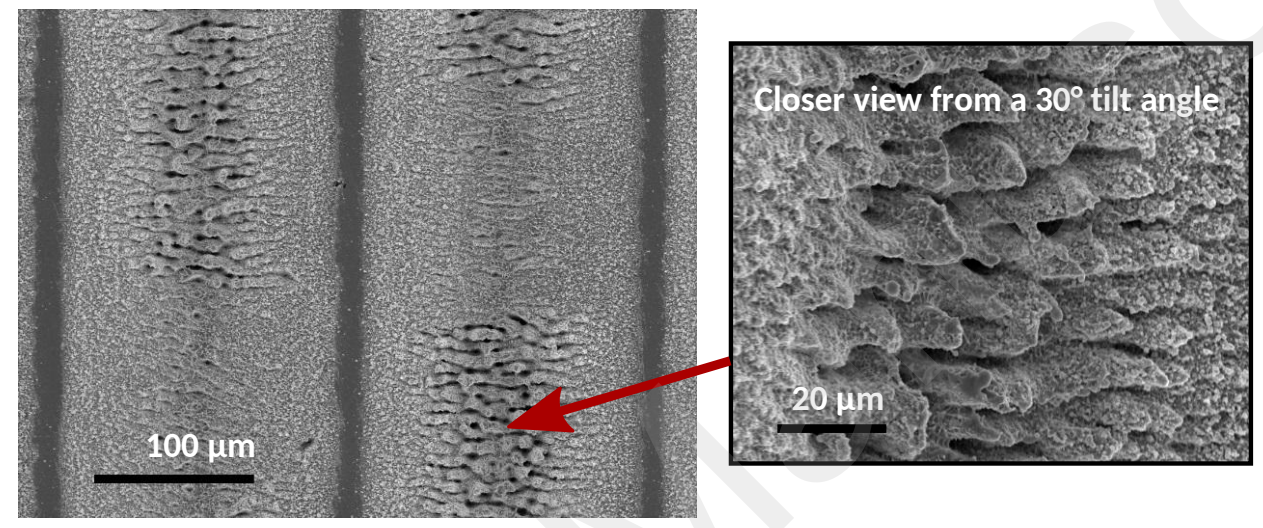

Figure 14. Scanning electron micrographs of a grooved pattern of wavelength $\lambda=180 \mu \mathrm{m}$, showing rough zones resulting from laser engraving, likely to contribute to the ejection process.

Most targets exhibit several grooves, between 8 and 17, depending on the wavelength $\lambda$. Ejection from a single groove, as illustrated in Figure 13 , is relatively non-uniform along the groove direction. Thus, in the case of multiple parallel grooves, piling up the ejecta sheets in the shadowgraphy direction forms a front delimited by the fastest particles of all sheets, so that the velocity of this front should be considered as an upper bound of jet tip velocity.

Finally, unexpectedly fast particles may also be due to the surface roughness at the bottom of the grooves, which exhibit locally narrow pits resulting from laser engraving, deeper and more widespread in the patterns of largest wavelengths (Figure 14). Such sharp geometrical defects are likely to contribute to the ejection process, generating very fast ejecta ahead of the main cloud. This may explain why both $v_{\mathrm{jt}}$ and $v_{99 \%}$ are unexpectedly high for $\lambda=180 \mu \mathrm{m}$ in Figure 12.

In addition to ejection velocity, RMI-based analyses also provide the areal mass of ejecta, which basically increases with $k \eta$ and $\lambda$, in accordance with the early, phenomenological prescription assuming that this mass would be proportional to the "missing mass" in the surface defect 37, 38. Next, our mass measurements are compared with the predictions of four RMI models, proposed by Cherne et al. 27 and Georgievskaya and Raevsky [39]. The first expression is Eq. 3 in Ref [27, referred to as the bubble shape source model:

$$
M_{\mathrm{ej}}(t)=M_{0} \log \left(1+\frac{t}{\tau}\right)
$$

where $M_{0}=\frac{\rho_{\text {liq }} \lambda}{3 \pi}, \tau=\frac{\lambda}{3 \pi\left|\dot{\eta}_{0}^{b}\right|}, \rho_{\text {liq }}=6.987 \cdot 10^{3} \mathrm{~kg} / \mathrm{m}^{3}$ is the density of liquid tin $[29]$ and $\left|\dot{\eta}_{0}^{s, b}\right|=F_{l} F_{n l}^{s, b} v_{\mathrm{fs}}$ are respectively spike and bubble initial relative velocities, in accordance with Ref. 24 notations and expressions. The second expression is Eq. 6 in the same Ref. 27, where a quadratic bubble shape evolution is assumed and the growth rate is modified by a $\beta$ factor:

$$
M_{\mathrm{ej}}(t)=\frac{2}{3} M_{0} \log \left(1+\frac{t}{\beta \tau}\right)
$$


Table II. Values of the main input parameters used for the comparison of our mass measurements with the predictions of RMI-based ejecta source models [27, 39].

\begin{tabular}{|c|c|c|c|c|c|c|c|}
\hline Id & $\left|\dot{\eta}_{0}^{s}\right|[\mathrm{km} / \mathrm{s}]$ & $\left|\dot{\eta}_{0}^{b}\right|[\mathrm{km} / \mathrm{s}]$ & $\tau[\mathrm{ns}]$ & $\beta$ & $\Delta t[\mathrm{~ns}]$ & $\beta+\frac{\Delta t}{\tau}$ & $\nu[\mathrm{ns}]$ \\
\hline Sn06 & 0.67 & 0.65 & 9.8 & 1.56 & 5.1 & 2.08 & 8.3 \\
\hline Sn08 & 1.75 & 2.01 & 3.2 & 1.66 & 8.6 & 4.38 & 3.1 \\
\hline Sn10 & 0.75 & 0.73 & 17.6 & 1.56 & 10.1 & 2.13 & 14.9 \\
\hline Sn14 & 0.75 & 0.73 & 17.7 & 1.56 & 9.4 & 2.10 & 14.9 \\
\hline Sn16 & 1.02 & 1.00 & 6.4 & 1.57 & 5.1 & 2.37 & 5.5 \\
\hline Sn17 & 0.98 & 0.97 & 6.5 & 1.57 & 5.5 & 2.40 & 5.6 \\
\hline Sn21 & 0.93 & 0.91 & 11.2 & 1.57 & 7.9 & 2.27 & 9.5 \\
\hline Sn25 & 0.69 & 0.67 & 9.4 & 1.56 & 4.8 & 2.07 & 8.0 \\
\hline Sn27 & 0.78 & 0.76 & 25.4 & 1.56 & 13.6 & 2.10 & 21.4 \\
\hline Sn32 & 0.82 & 0.80 & 24.3 & 1.56 & 12.7 & 2.09 & 20.5 \\
\hline Sn12 & 0.72 & 0.70 & 9.1 & 1.56 & 4.8 & 2.10 & 7.7 \\
\hline Sn30 & 0.74 & 0.72 & 8.8 & 1.56 & 4.9 & 2.11 & 7.5 \\
\hline
\end{tabular}
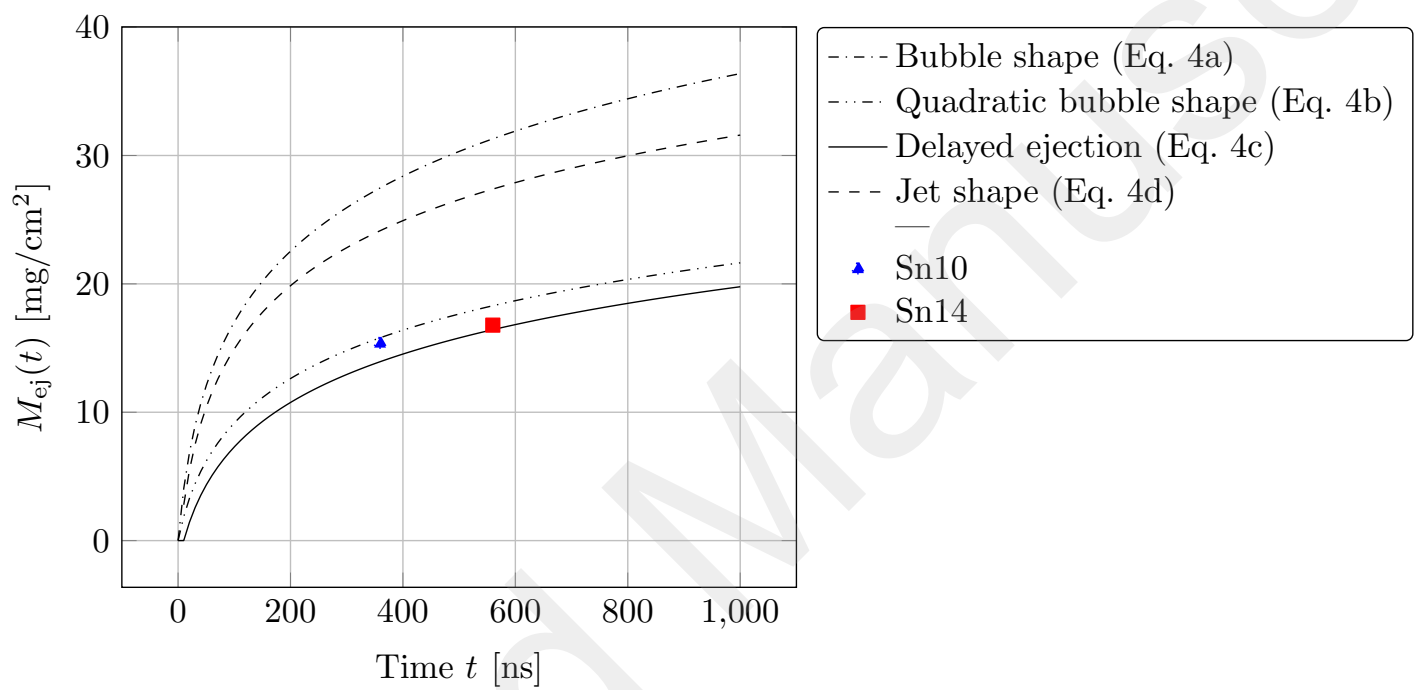

Figure 15. $M_{\mathrm{ej}}(t)$ vs. RMI as a function of time for experiments $\operatorname{Sn} 10\left(\lambda=120 \mu \mathrm{m}, k \eta=0.42, t_{\mathrm{rad}}=359 \mathrm{~ns}\right)$ and $\mathrm{Sn} 14$ $\left(\lambda=120 \mu \mathrm{m}, k \eta=0.40, t_{\mathrm{rad}}=560 \mathrm{~ns}\right)$.

where $\beta=1+\frac{1}{\sqrt{3}} \frac{F_{n l}^{b}}{F_{n l}^{s}}$. Eq. $4 \mathrm{~b}$ will be referred to as the quadratic bubble shape model. The third expression is Eq. 9 in Ref. 27, where initial bubble velocity is assumed to be 0 and a delay time $\Delta t$ is introduced between shock breakout and ejecta production, which affects the growth rate:

$$
M_{\mathrm{ej}}(t)=\frac{2}{3} M_{0} \log \left(1+\frac{t-\Delta t}{\left(\beta+\frac{\Delta t}{\tau}\right) \tau}\right)
$$

where $\Delta t=\exp \left(\frac{3}{2} k \eta F_{l}\right)-1$. Eq. $4 \mathrm{c}$ will be referred to as the delayed ejection model.

Finally, the fourth expression, taken from Ref. 39, involves a mass parameter $M_{\mathrm{GR}}$ (approximately equal to $3 / 4 M_{0}$ as compared with Eq. 4a above) and a growth time $\nu$ depending on the asymptotic spike velocity instead of the initial bubble velocity:

$$
M_{\mathrm{ej}}(t)=M_{\mathrm{GR}} \frac{\frac{t}{\nu}\left[\log \left(1+\frac{t}{\nu}\right)-k \eta\right]}{\frac{t}{\nu}+\log \left(1+\frac{t}{\nu}\right)} \underset{t \rightarrow+\infty}{\sim} M_{\mathrm{GR}} \log \left(1+\frac{t}{\nu}\right)
$$

where $M_{\mathrm{GR}}=\frac{\rho_{0} \lambda}{4 \pi}$ and $\nu=\frac{\lambda}{2 \pi \dot{\eta}_{\infty}^{s}}$. Eq. $4 \mathrm{~d}$ will be referred to as the jet shape model.

The values of the main input parameters in the above equations calculated for each of our laser shock experiments are listed in Table II. 
In Figure 15, the temporal evolution of the ejected mass measured at two successive times after shock breakout is compared with the mass inferred from these different equations. Experimental values are found to lie in the correct range (although low), in better agreement with Eq. 9 in Ref. 27] accounting for quadratic bubble shape and delay time.

(a) Bubble shape (Eq. 4a)

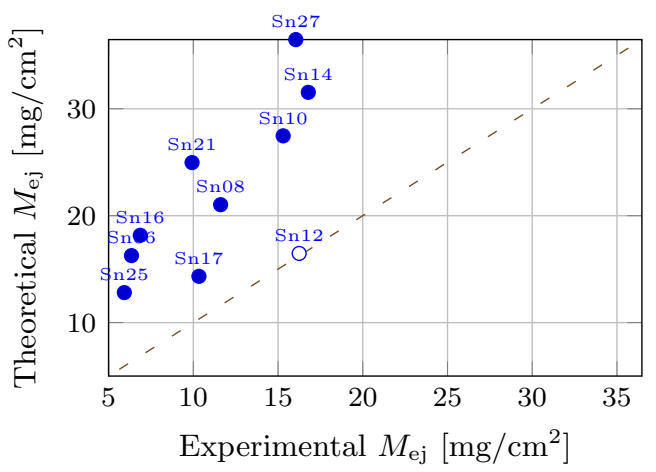

(c) Delayed ejection (Eq. 4c)

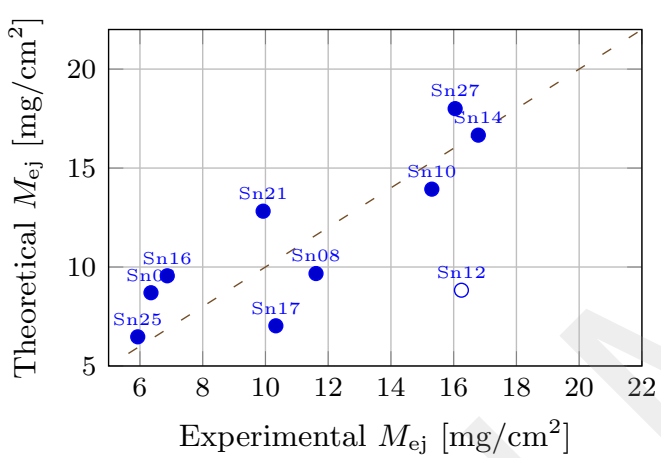

(b) Quadratic bubble shape (Eq. 4b)

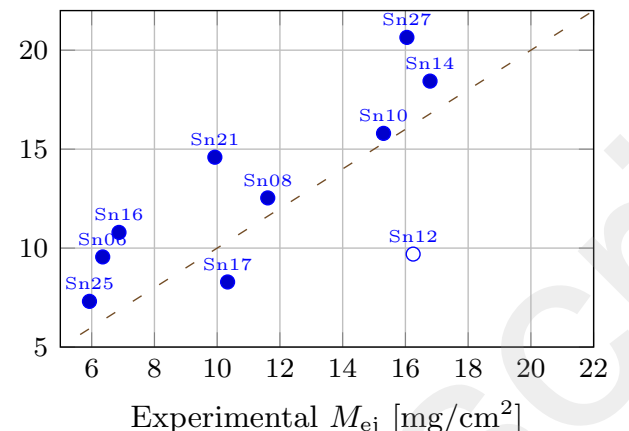

(d) Jet shape (Eq. 4d)

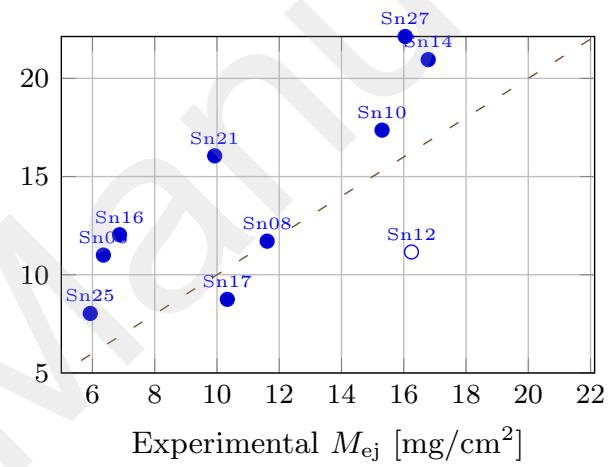

Figure 16. Comparison of the measured masses with those predicted by some RMI models. The dotted lines represent a perfect match between theory and experiment. Note that experiment Sn12 involves a single groove, which does not suit the RMI description. (a) Bubble shape (Eq. 4a), (b) Quadratic bubble shape (Eq. 4b), (c) Delayed ejection (Eq. 4c), (d) Jet shape (Eq. 4d).

An overall comparison of the ejected mass inferred from our radiographs with the predictions of the four models above is plotted in Figure 16, where the blue lines correspond to a perfect match between experiment and theory. Again, the order of magnitude is fairly consistent, although in most cases, measured masses lie below model predictions. The experiment Sn12 stands out because this particular target presented only one single groove, so that inadequate boundary conditions deviate from the RMI framework. Indeed, strong influences of edge effects on ejecta density [40, jet velocity 25 and particle size distribution 32 have been reported in the past. Going into more details, most measured masses are lower than expected from bubble shape (Eq. 4a), quadratic bubble shape (Eq. 4b), and jet shape (Eq. 4d) models (Figure 16a, b and d). A better overall agreement is found with delayed ejection model (Eq. 4c) (Figure 16c). The introduction of the quadratic bubble shape (using the $\beta$ factor) seems well suited to our experimental conditions involving rather small perturbation wavelengths, in (or near) a linear regime, with free surface velocities of a few $\mathrm{km} / \mathrm{s}$ and a growth duration $\tau$ between 5 and $25 \mathrm{~ns}$. Thus, the introduction of the delay time $\Delta t$ seems to fit our masses better, which demonstrates the relevance of including this parameter in modeling.

A promising way to investigate microjetting is Molecular Dynamics (MD) simulation [7, 11, 41. However, despite fast progress in computational capabilities, spatial and temporal scales in this approach are still limited to nm- and ps orders, respectively. Nevertheless, authors can invoke the scale invariance predicted by the RMI theory (since the above models do not involve dimensional parameters) to extrapolate their results (for a given $k \eta$ ) to the $\mu \mathrm{m}$ (or mm) dimensions involved in practical applications. Such scale invariance, which was not evidenced experimentally yet, can be tested to some extent in some of our experiments. Thus, in shots Sn27 and Sn10, which present the same shape factor $(k \eta \approx 0.41)$ but different groove depths and wavelengths, the main ejection features and velocities are fairly similar (Figure 8). 


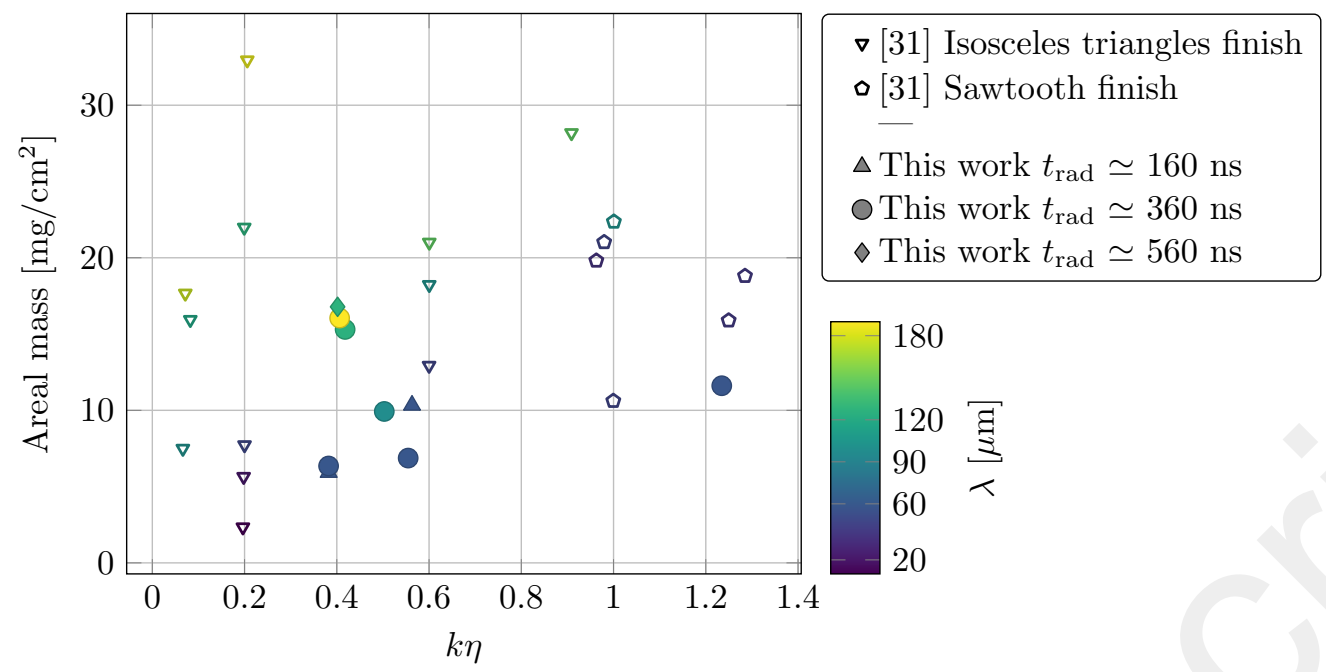

Figure 17. Comparison between the areal mass ejected by laser-driven shocks and those produced by high-explosive experiments (from Ref. 31) over the same range of shape factor $k \eta$.

To date, most experimental data on shock-induced ejection were reported under explosive-driven loading, e.g. Ref. 31. Key differences between these experiments and our laser-driven shocks include (i) a much shorter duration (by about two orders of magnitude) of the laser-driven pressure pulse, leading to a major contribution of the incident release wave following the shock front and extensive cavitation in the bulk specimen (see Section IIIC) ; (ii) a significantly higher loading pressure, expected to produce full melting on release ; (iii) measurements over shorter time ranges, probing earlier stages of the jetting process ; (iv) very different experimental diagnostics, especially to determine the ejected mass ; and (v) smaller specimen dimensions, with surface perturbations of sinusoidal shape in our case (while many explosive experiments involve chevron shapes). To explore the possible effects of such differences on the ejection process, Figure 17] shows a comparison of the mass densities inferred from our radiographs with similar areal densities reported under explosive-driven loading [31. Although the former tend to be somewhat lower than the latter, within the (substantial) scatter, a correct overall consistency is observed between both approaches over the same range of perturbation depths and wavelengths. Thus, both experimental techniques appear complementary in probing ejecta physics.

\section{CONCLUSION}

In our ongoing investigation of material ejection from surface defects under laser driven shock loading, we have used our recent ps-laser generated X-ray probe to perform a parametric study of microjetting from periodic grooves of quasi-sinusoidal shape in fully melted-on-release tin. Perturbations correspond to a linear (or almost linear) instability growth regime $(k \eta \lesssim 1)$, with grooves depths (peak-to-valley) ranging from $8 \mu \mathrm{m}$ to $24 \mu \mathrm{m}$. Complementary diagnostics are combined, namely PDV, optical shadowgraphy, and X-ray radiography, to provide ejection ("spike") velocities and estimate areal masses of ejecta. A second-order-derivative criterion is proposed for a more objective determination of the free surface location during ejection. Within the scatter, a fair consistency is found between our results and similar data reported under explosive-driven loading, despite major differences, both physical (drastically different durations of the pressure load) and technical (different diagnostics) between both experimental approaches. Furthermore, comparisons with RMI-based models, including recent time-dependent evolutions, are found to be fully relevant, for both ejection velocities and areal mass densities.

Among the improvements to be addressed in the future, two major issues are the planarity of the shock front (related to the spatial uniformity of the laser energy over the irradiated spot), and the repeatability and homogeneity of the laser-generated X-ray probe.

\section{ACKNOWLEDGMENTS}

The access to the Laboratoire pour l'Utilisation des Lasers Intenses (LULI) facility was allocated by the Institut Laser Plasma (ILP, FR \#2707). We thank all the NANO2000 staff for their technical support. 


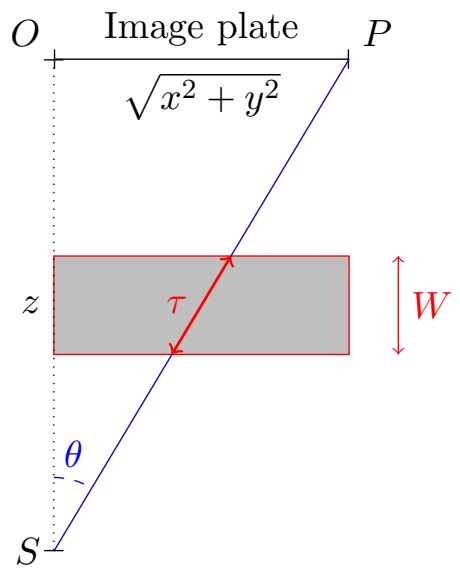

Figure 18. Punctual source and parallax corrections.

\section{DATA AVAILABILITY STATEMENT}

The data that support the findings of this study are available within the article. Additional details can be provided by the corresponding author upon reasonable request.

\section{Appendix A: Punctual source and parallax corrections}

Assuming that X-ray source is a point, the irradiance on the image plate is:

$$
E(r)=E_{0} \frac{\cos (\theta)}{\sqrt{O S^{2}+O P^{2}}}=E_{0} \frac{Z_{i p}}{\left(Z_{i p}^{2}+x^{2}+y^{2}\right)^{3 / 2}}
$$

with $Z_{i p} \approx 11 \mathrm{~cm}$ is the distance between source and image plate (Figure 18). So the image is scaled by $\left(Z_{i p}^{2}+x^{2}+y^{2}\right)^{3 / 2}$. The maximum correction at the ejecta outer edges is below $13 \%$.

As introduced in Section IIIC, the equivalent thickness $\ell$ is the width of a standard tin foil whose mass (per unit square, implicitly) is equivalent to the mass of the cloud along $z$-axis. Considering our punctual X-ray source, parallax and subsequent attenuation should be accounted for in the analysis (Figure 18). Thus, the equivalent thickness along $z$-axis is $\ell=\tau \cdot \cos (\theta)$. The minimum of $\cos (\theta)$ is $95 \%$ at the outer ejecta edges.

\section{Appendix B: Locating the free surface using a second derivative criterion}

Total ejecta mass $M_{\mathrm{ej}}$ in the jets is known to be very sensitive to $x_{\mathrm{fs}}$, the location of the free surface (see Figure 7). To improve both accuracy and objectivity of the determination of $x_{\mathrm{fs}}$, we resort to the following procedure.

Figure 19 (top) shows a schematic variation of density along the jetting direction. Within the jets expanding to the right, ahead of the free surface, the mean density is typically about $4 \% \rho_{0}$ in our experiments (e.g. experiment Sn27), where $\rho_{0}$ is the density of solid tin. Inside the bulk beneath the free surface, density is much higher, but still lower than $\rho_{0}$ due to melting, bubble growth and cavitation waves. The interface between both regions is not sharp since parallax and edge effects may affect X-ray measurements. However, ps-laser X-ray source limits the width of this transition, typically $30 \mu \mathrm{m}$ (e.g. radiograph Sn14).

Figure 19 (bottom) shows the second derivative of the areal mass $\frac{\mathrm{d}^{2} M_{\mathrm{S}}(x)}{\mathrm{d} x^{2}}$, i.e. the first derivative of the density, in the ideal case pictured above. We propose to use the minimum in this curve as a criterion to determine the location $\hat{x}_{\mathrm{fs}}$ of the free surface. In real experiments, variations present parasitic oscillations, but this minimum can usually be detected (see Figure 7). Note that this location is close to that inferred from the edge of the grooved zone.

[1] J. Yeager, P. Bowden, D. Guildenbecher, and J. Olles, J. Appl. Phys 122, 35901 (2017). 

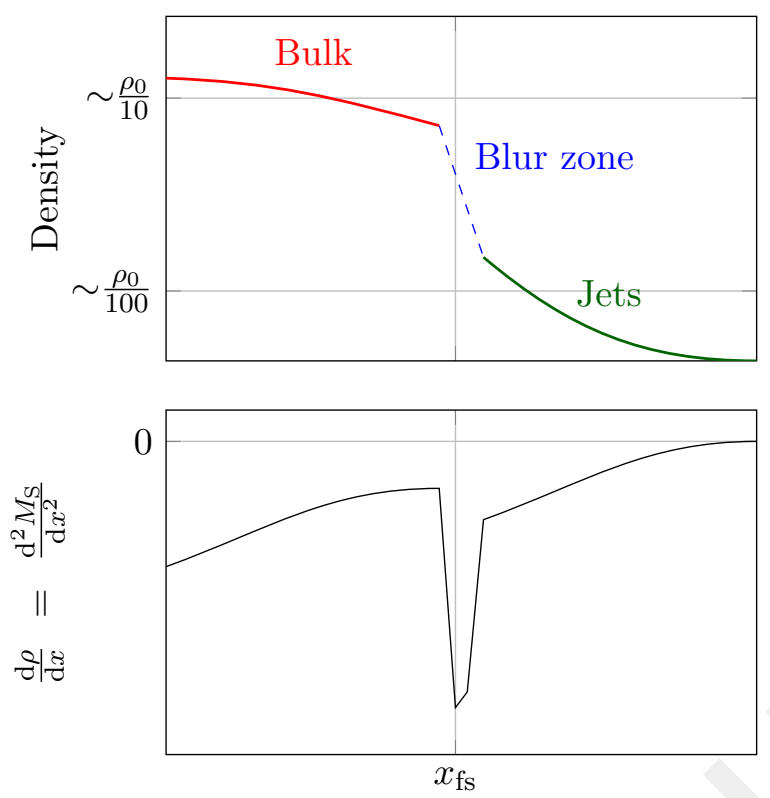

Distance $x$ along the expansion direction

Figure 19. Schematic evolution of ejecta density and its derivative.

[2] P. Bowden, J. Yeager, D. Guildenbecher, J. Olles, J. Hashem, and A. Schmalzer, AIP Conf. Proc., AIP Conf. Proc. 1979, 100006 (2018)

[3] D. Eder, A. Fisher, A. Koniges, and N. Masters, Nucl. Fusion 53, 113037 (2013).

[4] N. Masters, A. Fisher, D. Kalantar, J. Stölken, C. Smith, R. Vignes, S. Burns, T. Doeppner, A. Kritcher, and H.-S. Park, J. Phys. Conf. Ser. 717, 12108 (2016)

[5] W. T. Buttler, R. J. Williams, and F. M. Najjar, Journal of Dynamic Behavior of Materials 3, 151 (2017)

[6] G. Chu, T. Xi, M. Yu, W. Fan, Y. Zhao, M. Shui, W. He, T. Zhang, B. Zhang, Y. Wu, W. Zhou, L. Cao, J. Xin, and Y. Gu, Rev. Sci. Instrum. 89, 115106 (2018)

[7] B. Wu, F. Wu, Y. Zhu, P. Wang, A. He, and H. Wu, AIP Adv. 8, 045002 (2018)

[8] J. Xin, A. He, W. Liu, G. Chu, M. Yu, W. Fan, Y. Wu, T. Xi, M. Shui, Y. Zhao, P. Wang, Y. Gu, and W. He, J. Micromech. Microeng. 29, 095011 (2019)

[9] R. J. R. Williams, AIP Conf. Proc. 1979, 080015 (2018),

[10] A. M. Saunders, S. J. Ali, H. S. Park, J. Eggert, F. Najjar, C. Huntington, T. Haxhilmali, B. Morgan, Y. .Ping, and H. G. Rinderknecht, Development of High Power Laser Platforms to Study Metal Ejecta Interactions, Tech. Rep. LLNL-PROC783445 (Lawrence Livermore National Laboratories, 2019).

[11] O. Durand, S. Jaouen, L. Soulard, O. Heuzé, and L. Colombet, J. Appl. Phys. 122, 135107 (2017).

[12] O. Durand, L. Soulard, L. Colombet, and R. Prat, J. Appl. Phys. 127, 175901 (2020).

[13] J. E. Hammerberg, W. T. Buttler, A. Llobet, C. Morris, J. Goett, R. Manzanares, A. Saunders, D. Schmidt, A. Tainter, W. Vogan-McNeil, and C. Wilde, AIP Conf. Proc. 1979, 080006 (2018)

[14] I. L. Tregillis and A. Koskelo, Journal of Verification, Validation and Uncertainty Quantification 4, 10.1115/1.4045747 (2020), 041004.

[15] T. de Rességuier, E. Lescoute, A. Sollier, G. Prudhomme, and P. Mercier, J. Appl. Phys. 115, 043525 (2014),

[16] C. Roland, T. de Rességuier, A. Sollier, E. Lescoute, D. Loison, and L. Soulard, Journal of Dynamic behavior of Materials 3, 156 (2017).

[17] T. de Rességuier, G. Prudhomme, C. Roland, E. Brambrink, D. Loison, B. Jodar, E. Lescoute, and A. Sollier, J. Appl. Phys 124, 065106 (2018)

[18] T. de Rességuier, C. Roland, E. Lescoute, A. Sollier, D. Loison, L. Berthe, G. Prudhomme, and P. Mercier, AIP Conf. Proc. 1793, 100025 (2017).

[19] A. Sollier and E. Lescoute, Int. J. Impact Eng. 136, 103429 (2020)

[20] A. Sollier and E. Lescoute, AIP Conf. Proc. (2020), to be published.

[21] C. Roland, T. de Rességuier, A. Sollier, E. Lescoute, D. Tandiang, M. Toulminet, and L. Soulard, AIP Conf. Proc. 1979, $080012(2018)$

[22] G. Prudhomme, J.-E. Franzkowiak, T. de Rességuier, E. Brambrink, C. Roland, D. Loison, E. Lescoute, and A. Sollier, AIP Conf. Proc. 1979, 080010 (2018). 
[23] T. de Rességuier, G. Prudhomme, C. Roland, A. Sollier, E. Lescoute, D. Loison, and E. Brambrink, AIP Conf. Proc. (2020), to be published.

[24] W. T. Buttler, D. M. Oró, D. L. Preston, K. O. Mikaelian, F. J. Cherne, R. S. Hixson, F. G. Mariam, C. Morris, J. B. Stone, G. Terrones, and D. Tupa, J. Fluid Mech. 703, 60 (2012).

[25] T. de Rességuier, C. Roland, G. Prudhomme, E. Lescoute, D. Loison, and P. Mercier, J. Appl. Phys. 119, 185108 (2016).

[26] M. O. Mikaelian, Phys. Rev. A 31, 410 (1985)

[27] F. J. Cherne, J. E. Hammerberg, M. J. Andrews, V. Karkhanis, and P. Ramaprabhu, J. Appl. Phys. 118, 185901 (2015)

[28] C. Mabire, Transformation polymorphique et fusion de l'étain sous choc dans la gamme 0-100 GPa. Etude expérimentale et modélisation., $\mathrm{PhD}$ thesis, Université de Poitiers (1999).

[29] C. Mabire and P.-L. Hereil, in Shock compression of condensed matter - 1999, pts 1 and 2, AIP Conf. Proc., Vol. 505, edited by M. D. Furnish, L. C. Chhabildas, and R. S. Hixson (2000) pp. 93-96.

[30] D. Loison, T. de Rességuier, A. Dragon, P. Mercier, J. Benier, G. Deloison, E. Lescoute, and A. Sollier, J. Appl. Phys. 112, $8(2012)$

[31] S. K. Monfared, D. M. Oró, M. Grover, J. E. Hammerberg, B. M. LaLone, C. L. Pack, M. M. Schauer, G. D. Stevens, J. B. Stone, W. D. Turley, and W. T. Buttler, J. Appl. Phys. 116, 063504 (2014).

[32] M. M. Schauer, W. T. Buttler, D. K. Frayer, M. Grover, B. M. LaLone, S. K. Monfared, D. S. Sorenson, G. D. Stevens, and W. D. Turley, Journal of Dynamic Behavior of Materials 3, 10.1007/s40870-017-0111-9 (2017).

[33] Q. Zhang, Physical review letters 81, 3391 (1998).

[34] G. Dimonte, G. Terrones, F. J. Cherne, and P. Ramaprabhu, J. Appl. Phys. 113, 19 (2013).

[35] J. E. Hammerberg, F. J. Cherne, M. J. Andrews, P. Ramaprabhu, and W. T. Buttler, Journal of Dynamic Behavior of Materials 3, 316 (2017).

[36] V. Karkhanis, P. Ramaprabhu, F. J. Cherne, J. E. Hammerberg, and M. J. Andrews, J. Appl. Phys. 123, 025902 (2018)

[37] J. R. Asay and L. D. Bertholf, A model for estimating the effects of surface roughness on mass ejection from shocked materials, Tech. Rep. SAND78-1256 (Sandia Laboratories, 1978).

[38] M. B. Zellner, G. Dimonte, T. C. Germann, J. E. Hammerberg, P. A. Rigg, G. D. Stevens, W. D. Turley, and W. T. Buttler, in Shock Compression of Condensed Matter - 2009, Pts 1 and 2, AIP Conf. Proc., Vol. 1195, edited by M. L. Elert, W. T. Buttler, M. D. Furnish, W. W. Anderson, and W. G. Proud (2009) pp. 1047-1050.

[39] A. Georgievskaya and V. A. Raevsky, AIP Conf. Proc. 1426, 1007 (2012).

[40] S. K. Monfared, W. T. Buttler, D. K. Frayer, M. Grover, B. M. LaLone, G. D. Stevens, J. B. Stone, W. D. Turley, and M. M. Schauer, J. Appl. Phys. 117, 223105 (2015).

[41] O. Durand and L. Soulard, J. Appl. Phys. 117, 165903 (2015) 